\title{
Bloqueo de Pericapsular Nerve Group en cirugía de cadera. Experiencia analgésica perioperatoria
}

\section{Pericapsular Nerve Group blockade in hip surgery. Perioperative analgesic experience}

\author{
Luis Emilio Flores Cadena, * José Manuel Athié García ${ }^{\ddagger}$ \\ Citar como: Flores CLE, Athié GJM. Bloqueo de Pericapsular Nerve Group en cirugía de cadera. Experiencia \\ analgésica perioperatoria. Acta Med GA. 2021; 19 (4): 480-484. https://dx.doi.org/10.35366/102531
}

\section{Resumen}

La fractura de cadera es una entidad quirúrgica que se presenta principalmente en adultos mayores cuyas comorbilidades plantean un reto anestésico. Objetivo: Reportar la experiencia analgésica perioperatoria en pacientes operados de cirugía de cadera en el hospital Angeles Mocel. Material y métodos: Presentamos un estudio observacional, descriptivo y prospectivo de 14 casos intervenidos en los que se realizó bloqueo del grupo de nervios pericapsulares (Pericapsular Nerve Group, PENG por sus siglas en inglés) ecoguiado con $30 \mathrm{~mL}$ de ropivacaína $0.5 \%$ y en quienes se evaluó dolor referido en reposo y movimiento 20 minutos después de la administración y durante las primeras 10 horas. Resultados: Tras 20 minutos de la administración, el dolor en reposo disminuyó $25.6 \%$ y a la movilización activa $39.58 \%$ en escala ENA respecto al basal previo al bloqueo. Los pacientes se mantuvieron con dolor leve durante las primeras 10 horas posteriores. Conclusiones: El empleo del bloqueo de PENG es una alternativa eficaz para el control de dolor perioperatorio en casos con cirugía de cadera, brindando analgesia hasta por 10 horas y permitiendo la movilización temprana e inicio de rehabilitación.

Palabras clave: Bloqueo de PENG, cirugía de cadera, analgesia para cirugía de cadera.

\section{INTRODUCCIÓN}

La fractura de cadera es una urgencia ortopédica de resolución principalmente quirúrgica. ${ }^{1}$ Si bien se presenta a cualquier edad como resultado de accidentes, el mayor número de casos se encuentra entre la po-

\section{Abstract}

Hip fractures are surgical entities that are mostly present in elderly individuals with frequently challenging comorbidities. Objective: Report our perioperative analgesic experience in the Angeles Mocel Hospital in patients undergoing hip surgery. Material and methods: We present an observational, descriptive, and prospective study in 14 patients in which ECO-guided PENG block was administered with $30 \mathrm{~mL}, 0.5 \%$ ropivacaine. Referred pain was assessed at rest and at active movement 20 minutes and during the first ten hours after block administration. Results: 20 minutes after block administration, resting referred pain diminished $25.6 \%$ points (NRS scale) and active movement referred pain diminished $39.58 \%$ according to basal pre-block referral. Patient pain remained mild during the subsequent 10 hours. Conclusions: The PENG block is an effective alternative for perioperative pain management in patients undergoing hip fracture surgery with adequate pain inhibition and facilitated movement and early rehabilitation in the first postsurgical hours.

Keywords: PENG blockade, hip surgery, analgesia for hip surgery.

blación geriátrica, con comorbilidades degenerativas y en muchas ocasiones en situación de fragilidad, lo que complica su pronóstico y hace el manejo perioperatorio más complejo. ${ }^{2}$

Se estima que en México 25\% de la población adulta padece osteopenia $y$, conforme la tendencia actual de

\footnotetext{
* Médico Residente de Anestesiología. Alumno de la Facultad Mexicana de Medicina de la Universidad La Salle.

₹ Titular de Cátedra de Anestesiología.
}

Correspondencia:

Luis Emilio Flores Cadena

Correo electrónico: osasgoul@hotmail.co.uk

Aceptado: 09-11-2020. 
envejecimiento poblacional se mantenga, este número aumentará ${ }^{3}$ así como la prevalencia de enfermedades crónicas, que nos obliguen a un abordaje interdisciplinario enfocado a limitar las complicaciones perioperatorias y favorecer protocolos de recuperación temprana. ${ }^{4}$

En este contexto, las técnicas anestésicas multimodales de mínima invasión con esquemas optimizados para la disminución del consumo de opioides y sus efectos adversos cobran relevancia. ${ }^{5}$ Una técnica emergente propuesta es el bloqueo del grupo de nervios pericapsulares de la cadera

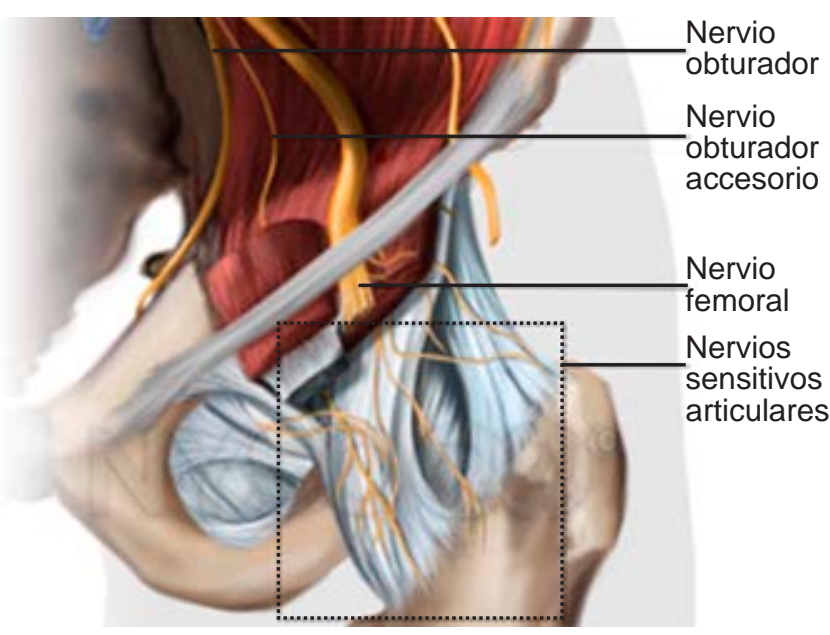

Figura 1: Inervación habitual de la cápsula anterior de la cadera. Modificada de: https://www.nysora.com/news/the-hipblock-new-addition-to-nysoras-web-app/
(Pericapsular Nerve Group, PENG por sus siglas en inglés), descrito a finales de 2018 por los Doctores Girón-Arango y Peng ${ }^{6}$ basados en el estudio anatómico del Dr. Short, en el que se demostró que los nervios femoral, obturador y obturador accesorio juegan un papel predominante en la inervación de la articulación de la cadera (Figura 1), y que identificó las referencias anatómicas que facilitan el abordaje ecoguiado (Figura 2). ${ }^{7}$

El objetivo de este estudio es utilizar la técnica descrita de bloqueo de PENG como coadyuvante a la anestesia neuroaxial durante el periodo perioperatorio, así como analizar el impacto analgésico en los pacientes sometidos a cirugía de cadera.

\section{MATERIAL Y MÉTODOS}

Se realizó un estudio observacional, descriptivo y prospectivo en casos con cirugía de cadera en el Hospital Mocel del grupo Angeles, dentro del periodo comprendido entre el de 2 de mayo al 20 de septiembre de 2019. Se incluyeron en la muestra por conveniencia un total de 14 adultos (Tabla 1), previo consentimiento informado, los criterios de inclusión fueron una valoración de riesgo de acuerdo con la escala de la ASA de entre I y III, que tuvieran factura de cadera unilateral, que se encontraran con adecuado estado de conciencia y fueran capaces de comprender la escala numérica análoga (ENA).

Se consideraron como criterios de exclusión: contraindicación para manejo mediante anestesia neuroaxial, ASA igual o mayor a IV, con tratamiento anticoagulante
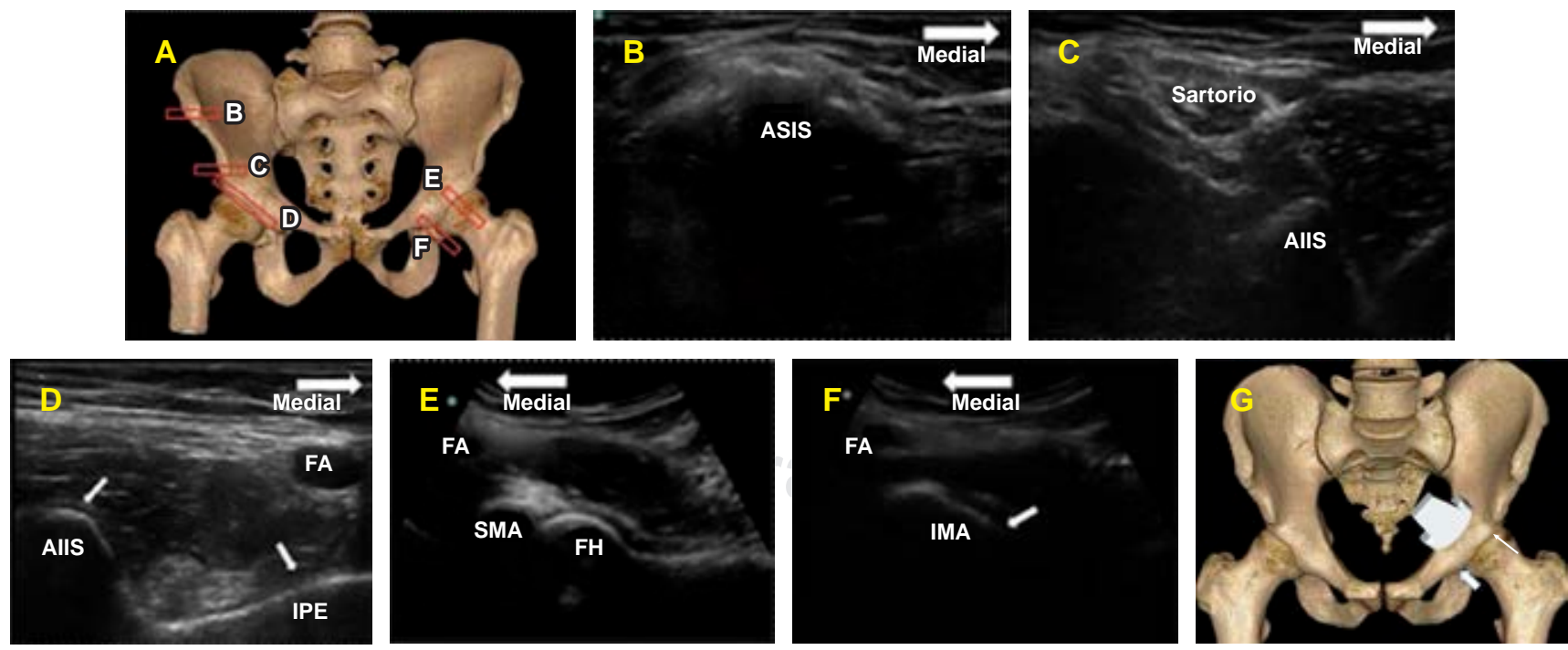

Figura 2: Localización y correlación de las estructuras anatómicas, sonoanatomía y posición del transductor. Modificada de: https://www.nysora.com/news/the-hip-block-new-addition-to-nysoras-web-app/. (Disponible hasta octubre de 2020 en el sitio oficial de nysora). 
Tabla 1: Serie de casos.

\begin{tabular}{ccccc}
$\begin{array}{c}\text { Edad } \\
\text { (años) }\end{array}$ & Sexo & EA & ASA & PQX \\
& & & & \\
95 & F & Izq. & III & Transtrocantérica \\
93 & F & Der. & III & Transtrocantérica \\
86 & M & Der. & II & Transtrocantérica \\
83 & M & Der. & II & Peritrocantérica \\
82 & F & Izq. & II & Peritrocantérica \\
80 & F & Izq. & II & Transtrocantérica \\
78 & M & Izq. & II & Peritrocantérica \\
71 & F & Der. & II & De cuello femoral \\
67 & F & Izq. & II & Peritrocantérica \\
65 & F & Der. & III & Transtrocantérica \\
61 & M & Izq. & II & Transtrocantérica \\
59 & F & Izq. & I & De cuello femoral \\
57 & F & Izq. & I & Transtrocantérica \\
42 & M & Der. & II & Peritrocantérica \\
\hline
\end{tabular}

$E A=$ extremidad afectada; $A S A=$ escala de riesgo de la Sociedad Americana de Anestesiólogos; PQX = localización del trazo de fractura.

Autoría propia.

o casos que rechazaran el procedimiento y que hubieran sido medicados con opioides.

Se valoró el dolor mediante la escala numérica análoga (ENA) en cuatro tiempos distintos: 1) al momento de la valoración preanestésica en reposo y a la movilización activa (basal); 2) a los 15 minutos, ambos posteriores al bloqueo de PENG en reposo y al posicionamiento en decúbito lateral; 3) cuatro horas después de la instalación del bloqueo espinal en reposo y a la movilización activa; y 4) 10 horas después del bloqueo de neuroeje en reposo y a la movilización activa y se obtuvo la media de cada valoración de ENA.

Técnica anestésica: en sala quirúrgica bajo monitoreo estándar II de la ASA, con oxígeno suplementario por puntas nasales a $2 \mathrm{~L} / \mathrm{min}$ en decúbito supino, se realizó asepsia de la región ilioinguinal ipsilateral a la fractura con clorhexidina $2 \%$, se utilizó un transductor lineal de alta frecuencia con protección estéril y gel de interfaz colocándose en plano transverso sobre la espina iliaca anteroinferior, se realizó rotación medial a $45^{\circ}$ aproximadamente hasta localizar la eminencia iliopúbica. Las estructuras a identificar son la arteria y vena femorales, los nervios femoral, obturador y obturador accesorio y el tendón del músculo psoas (Figura 3). Se utilizó una aguja Stimuplex de $22 \mathrm{G}$ de $100 \mathrm{~mm}$ ecogénica. Con técnica in plane, se depositaron $30 \mathrm{~mL}$ de ropivacaína $0.5 \%$, verificando su difusión en el plano musculofascial entre el tendón y la rama púbica. Quince minutos después, se colocó al paciente en decúbito lateral sobre el lado contrario a la fractura, se procedió al bloqueo neuroaxial con técnica subaracnoidea dosis única con aguja Whitacre 27 G ministrando 10 mg de bupivacaína isobárica. Durante el transanestésico, sólo se agregó paracetamol de 1 g y parecoxib de 40 mg como medicación analgésica y se continuó con horario. En caso de requerir analgesia adicional durante el periodo de evaluación, se utilizó tramadol $50 \mathrm{mg}$ como rescate.

\section{RESULTADOS}

Los pacientes incluidos es este estudio fueron 14 , de los cuales nueve (64\%) son mujeres y cinco (36\%) hombres, con una edad mínima de 42 años y edad máxima de 95 años, edad media de 72.78, la valoración de ASA fue I en dos pacientes, II en nueve pacientes, III en tres pacientes, la extremidad afectada izquierda se presentó en $57 \%$, y derecha en $43 \%$, siendo la cirugía más frecuente la reducción abierta y fijación interna (RAFI), en la evaluación de dolor con la escala de evaluación numérica análoga se obtuvieron las siguientes medias, la realizada previa al bloqueo (basal), en reposo se obtuvo una media de 6.9 y en movimiento 8.6, durante el transanestésico en reposo se obtuvo 1.7 y en movimiento 2.6, la evaluación a las cuatro horas posteriores en reposo fue de 1.3 y en movimiento 2.7 y a las 10 horas en reposo 2 y en movimiento 3.8, la medicación analgésica fue continuada con horario y no se requirieron rescates de opioides (Figura 4).

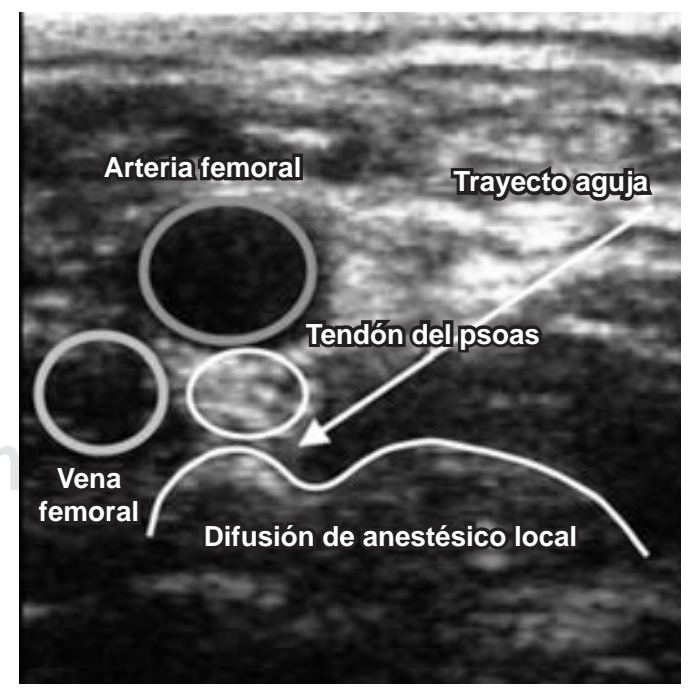

Figura 3: Trayecto de la aguja y difusión de anestésico local durante el abordaje. 
Figura 4: Resultados en escala numérica análoga (ENA) en los tiempos basal, transanestésico, a las 4 y 10 horas postoperatorias. Autoría propia.

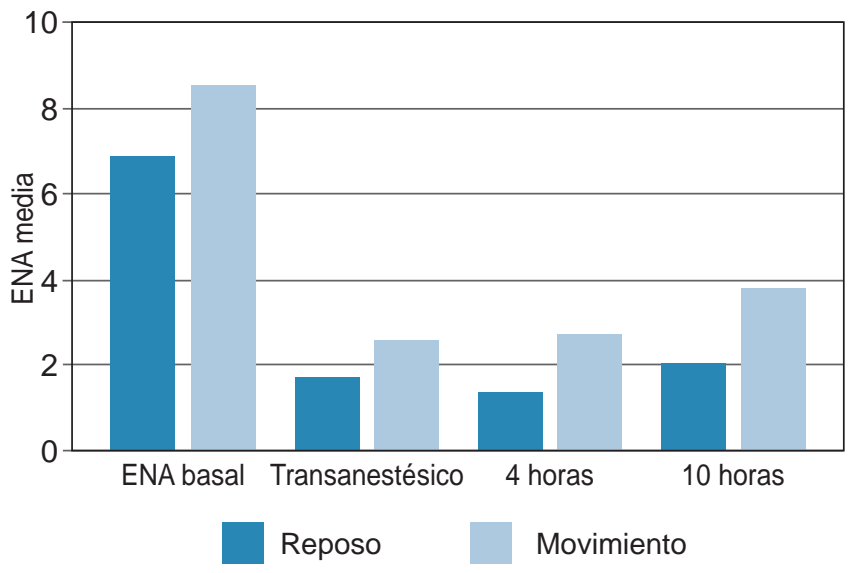

\section{DISCUSIÓN}

Los resultados obtenidos durante la realización de este trabajo son similares a los reportados en la bibliografía consultada. ${ }^{8}$ La utilización del bloqueo de PENG durante el perioperatorio se tradujo en una disminución promedio en escala de ENA en reposo de 5.2 puntos 20 minutos después de la administración del bloqueo, 5.6 puntos a las cuatro horas después de la cirugía y 4.9 puntos 10 horas después de finalizada la cirugía con un valor basal reportado de 6.9 puntos en promedio, ${ }^{9}$ y de $6,5.9$ y 4.8 (basal 8.6) puntos a la movilización en los mismos tiempos que en reposo, lo cual es similar a los estudios reportados. La duración de la analgesia (evaluada hasta las 10 horas postquirúrgicas) también es similar a lo reportado, manteniendo adecuado estado de confort y permitiendo tanto la deambulación como el inicio temprano de los protocolos de rehabilitación. ${ }^{10}$

Si bien la técnica descrita originalmente fue llevada a cabo con un transductor convexo, durante la realización de este trabajo se utilizó un transductor lineal de alta frecuencia sin que se hayan encontrado dificultades técnicas que impidieran la visualización adecuada de la sonoanatomía o el abordaje de las estructuras blanco. ${ }^{11}$ De igual manera, al considerar las recomendaciones publicadas por los Doctores Black y Chin encontramos que, en efecto, la localización de las estructuras se facilita al identificar de distal a proximal, y ubicando en toda ocasión el nervio femoral y los nervios accesorios, ${ }^{12}$ por lo que proponemos que esta técnica puede ser llevada a cabo sin dificultad con el transductor lineal.

En el artículo original, se describe la utilización de 20 $\mathrm{mL}$ de solución anestésica de bupivacaína $0.25 \%$ con epi- nefrina y en un solo caso ropivacaína $0.5 \%$ con epinefrina y dexametasona. ${ }^{13}$ En las otras investigaciones referidas, se evaluaron distintas concentraciones y volúmenes de anestésicos locales. Se especula que al aumentar el volumen del fármaco, la distribución dentro de los compartimentos capsulares e interfasciales abarca un mayor número de estructuras nerviosas, por lo que podría teóricamente usarse como bloqueo único. ${ }^{14}$ En esta publicación, decidimos utilizar ropivacaína y aumentar el volumen $(30 \mathrm{~mL})$ y la concentración $(0.5 \%)$, a fin de disminuir el tiempo de inicio de acción, aumentar la intensidad del bloqueo y el tiempo de efecto analgésico, lo cual resultó satisfactorio para ambos fines.

Algunos de los estudios consultados consideraron el empleo del bloqueo de PENG para diversas situaciones relacionadas con las fracturas de cadera. ${ }^{15} \mathrm{El}$ empleo durante el abordaje agudo puede ser beneficioso para facilitar la exploración y traslado intrahospitalario de los pacientes, disminuir el dolor a la movilización también es de utilidad cuando es necesario prolongar el tiempo hasta el tratamiento quirúrgico definitivo por razones médicas o administrativas, y disminuye las complicaciones asociadas con la inmovilización prolongada; el transanestésico se beneficia de un menor consumo de opioides sistémicos, además la combinación con técnicas de bloqueo continuo a través de catéter puede alargar el efecto de bloqueo de PENG hasta más de 100 horas. $^{16}$

\section{CONCLUSIÓN}

El bloqueo de PENG representa una opción adecuada como técnica coadyuvante en el periodo perioperatorio para cirugía de cadera. Puede impactar desde la atención en urgencias, facilitando la exploración y traslado intrahospitalario del paciente a estudios de imagen y al área quirúrgica; de igual manera, si la cirugía necesita diferirse por motivos clínicos o quirúrgicos, es útil para mejorar el confort y la movilidad, disminuyendo riesgo trombótico o de delirium. Durante el periodo transoperatorio, permite un abordaje anestésico regional con menor dolor a la movilización, lo cual también hace menos necesario el utilizar técnicas de anestesia general en casos con comorbilidades que incrementan el riesgo. También se abre la puerta a estudiar concentraciones y volúmenes de anestésico local que permitan utilizar el bloqueo como técnica anestésica única. Asimismo, disminuye los requerimientos de fármacos sistémicos, principalmente opioides. La analgesia postoperatoria es adecuada, duradera y, dado que no impide la movilidad, puede ser benéfica para el inicio de la movilización y rehabilitación temprana, disminuyendo el tiempo de estancia intrahospitalaria. Es 
recomendable realizar estudios con grupos mayores de casos que permitan aumentar el nivel de evidencia para estandarizar el uso de esta técnica en los protocolos de cirugía de cadera.

\section{REFERENCIAS}

1. Roy R, Agarwal G, Pradhan C, Kuanar D. Total postoperative analgesia for hip surgeries, PENG block with LFCN block. Reg Anesth Pain Med. 2019; 44(6): 684-690.

2. Doger EP, González LJ, King MAC. Fractura de cadera: un reto multidisciplinario. Reporte de caso de una víctima del sismo del 19 de septiembre de 2017. Rev Fac Med UNAM. 2019; 62(4): 24-29.

3. Hojer Karlsen AP, Geisler A, Petersen PL, Mathiesen O, Dahl JB. Postoperative pain treatment after total hip arthroplasty: a systematic review. Pain. 2015; 156(1): 8-30.

4. Yavuz F, Yasar E, Ali Taskaynatan M, Goktepe AS, Tan AK. Nerve block of articular branches of the obturator and femoral nerves for the treatment of hip joint pain. J Back Musculoskelet Rehabil. 2013; 26(1): 79-83.

5. Girón-Arango L, Peng PWH, Chin KJ, Brull R, Perlas A. Pericapsular nerve group (PENG) block for hip fracture. Reg Anesth Pain Med. 2018; 43(8): 859-863.

6. Zaragoza-Lemus G, Portela-Ortiz JM, Díaz-Guevara G. Bloqueo del grupo de nervios pericapsulares (PENG) para cirugía de cadera. Rev Mex Anest. 2020; 43(1): 69-72.

7. Acharya U, Lamsal R. Pericapsular nerve group block: an excellent option for analgesia for positional pain in hip fractures. Case Rep Anesthesiol. 2020; 2020: 1830136.

8. Pagano T, Scarpato F, Chicone G, Carbone D, Bussemi C, Albano F et al. Analgesic evaluation of ultrasound-guided Pericapsular Nerve Group (PENG) block for emergency hip surgery in fragile patients: a case series. J Arthroplasty. 2019; 1(1): 1-5.
9. Ahiskalioglu A, Aydin ME, Celik M, Ahiskalioglu EO, Tulgar S. Can high volume pericapsular nerve group (PENG) block act as a lumbar plexus block? J Clin Anesth. 2020; 61: 109650.

10. Aksu C, Cesur S, Kus A. Pericapsular nerve group (PENG) block: controversial points about anatomical differences. J Clin Anesth. 2020; 61: 109701.

11. Ueshima $H$, Otake $H$. Clinical experiences of pericapsular nerve group (PENG) block for hip surgery. J Clin Anesth. 2018; 51: 60-61.

12. Black ND, Chin KJ. Pericapsular nerve group (PENG) block: Comments and practical considerations. J Clin Anesth. 2019; 56: 143-144.

13. Orozco S, Muñoz D, Jaramillo S, Herrera AM. Pericapsular nerve group (PENG) block for perioperative pain control in hip arthroscopy. J Clin Anesth. 2020; 59: 3-4.

14. Bilal B, Oksuz G, Boran OF, Topak D, Dogar F. High volume pericapsular nerve group (PENG) block for acetabular fracture surgery: a new horizon for novel block. J Clin Anesth. 2020; 62: 109702.

15. Prado-Kittel C, Zumelzu-Sánchez P, Palma-Licandeo A, FaúndezLillo G, Ellenberg-Oyarce K, Jorquera-Adarme B. Bloqueo continuo del grupo de nervios pericapsular como analgesia para fractura de columna y pared posterior de acetábulo, reporte de caso y descripción de régimen de infusión para ampliar la cobertura analgésica a la región femoral distal. Rev Esp Anestesiol Reanim. 2020; 67(3): 159-162.

16. Molinelli M, Romero J, Uranga S, Bartolini J, Caputo N, Ariel L et al. Efecto analgésico del bloqueo peng (grupo de nervios pericapsulares) en pacientes con fractura de cadera. Rev Chil Anest. 2020; 49(1): 134-138.

Conflicto de intereses: Los autores declaran no tener ningún conflicto de intereses y no haber recibido patrocinio para la realización del trabajo. 